\title{
Dynamically Harmonized FT-ICR Cell with Specially Shaped Electrodes for Compensation of Inhomogeneity of the Magnetic Field. Computer Simulations of the Electric Field and Ion Motion Dynamics
}

\author{
Yury I. Kostyukevich, ${ }^{1}$ Gleb N. Vladimirov, ${ }^{1,2}$ Eugene N. Nikolaev ${ }^{1,2,3}$ \\ ${ }^{1}$ Institute for Energy Problems of Chemical Physics, Russian Academy of Sciences, Moscow, Russia \\ ${ }^{2}$ Emanuel Institute of Biochemical Physics, Russian Academy of Sciences, Moscow, Russia \\ ${ }^{3}$ Institute of Biomedical Chemistry, Russian Academy of Medical Sciences, Moscow, Russia
}

\begin{abstract}
The recently introduced ion trap for FT-ICR mass spectrometers with dynamic harmonization showed the highest resolving power ever achieved both for ions with moderate masses 500$1000 \mathrm{Da}$ (peptides) as well as ions with very high masses of up to $200 \mathrm{kDa}$ (proteins). Such results were obtained for superconducting magnets of very high homogeneity of the magnetic field. For magnets with lower homogeneity, the time of transient duration would be smaller. In superconducting magnets used in FT-ICR mass spectrometry the inhomogeneity of the magnetic field in its axial direction prevails over the inhomogeneity in other directions and should be considered as the main factor influencing the synchronic motion of the ion cloud. The inhomogeneity leads to a dependence of the cyclotron frequency from the amplitude of axial oscillation in the potential well of the ion trap. As a consequence, ions in an ion cloud become dephased, which leads to signal attenuation and decrease in the resolving power. Ion cyclotron frequency is also affected by the radial component of the electric field. Hence, by appropriately adjusting the electric field one can compensate the inhomogeneity of the magnetic field and align the cyclotron frequency in the whole range of amplitudes of z-oscillations. A method of magnetic field inhomogeneity compensation in a dynamically harmonized FT-ICR cell is presented, based on adding of extra electrodes into the cell shaped in such a way that the averaged electric field created by these electrodes produces a counter force to the forces caused by the inhomogeneous magnetic field.
\end{abstract}

Key words: FT-ICR, FTMS, Simulation

\section{Introduction}

Fourier transform ion cyclotron resonance mass-spectrometry is a well established powerful experimental technique for solving a wide range of problems in analytical chemistry and biochemistry, such as determination of the composition of

Correspondence to: E. N. Nikolaev; e-mail: ennikolaev@rambler.ru complex mixtures, identification of biological compounds, and accurate mass measurement [1-6].

The main part of the ICR mass spectrometer is a measuring cell, which is in fact the Penning ion trap in which ions are trapped by a combination of electric and magnetic fields. In order to measure the masses of the ions after they are trapped in the cell, cyclotron motion of the ions is excited by the rf field and the frequency of this 
motion is determined by measuring the current induced in the external electric circle connected to the detection electrodes of the cell. After the Fourier transform of this time domain signal one obtains its frequency spectrum and after calibration a mass spectrum.

The configuration of the electric field inside the ion trap strongly influences the analytical characteristics of the ICR mass spectrometer, its resolving power and mass accuracy [7, 8].

Recently performed supercomputer simulations of ion clouds motion in a Penning trap showed that the hyperbolic field is the best for achieving long duration of synchronous ion motion and obtaining high resolving power [9-11]. Making the electric field distribution inside the FT ICR cell close to the field in a hyperbolic cell we call cell harmonization.

Our approach to cell harmonization is based on the socalled dynamic harmonization of the electric field [12]. The cell field becomes hyperbolic after being averaged by the cyclotron motion. The principal design of such a cell was previously described in [12] and is presented in Figure 1a.

As described in [12] this cell is a cylinder segmented by curves along axial (magnetic field) direction

$$
\alpha=\frac{2 \pi}{N} n \pm b\left(1-\left(\frac{z}{a}\right)^{2}\right) ; n=0,1 \ldots, N-1
$$

Here the z-axial coordinate of the cell, $b=\frac{\pi}{N}-\frac{\pi}{60}, a-$ is half the length of the cell, $\alpha$ - the angle coordinate of a point on the curve, $N$ - number of electrodes of each type. The original experimentally tested ion trap with dynamic harmonization had eight segments with width decreasing to the center of the cell and eight grounded electrodes with width increasing to the center, four of which are divided into two segments, each of which belongs to either excitation or detection groups of electrodes. The trapping potential $\mathrm{V}$ is applied to first group of electrodes and to trapping electrodes. Other electrodes are grounded to DC voltage; rf voltages are applied via capacitors to excitation groups of electrodes and detection group electrodes are connected with each other and with preamplifier by capacitors of appropriate value of capacity.

The ion trap with dynamic harmonization showed the highest resolving power ever achieved on peptides and proteins [13]. The time of transient duration reaches $300 \mathrm{~s}$ and seems to be limited only by the vacuum inside the FT ICR cell and magnetic field inhomogeneity [14]. Such results were obtained on a solenoid magnet of high homogeneity (less than $1 \mathrm{ppm}$ of magnetic field in the central region [6 $\mathrm{cm}$ in diameter and $6 \mathrm{~cm}$ length]). In order to obtain a long time domain signal using the dynamically harmonized cell on the other systems, the magnetic field of their magnets should be corrected correspondingly. Among the systems of interest are FT ICR mass spectrometers on permanent magnets, with inhomogeneity of the magnetic field about $500 \mathrm{ppm}$ in a $1 \mathrm{~cm}^{3}$ cube [15], and on cryogenic (a)

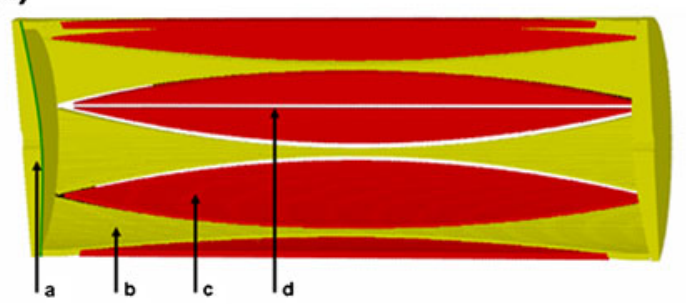

(c)

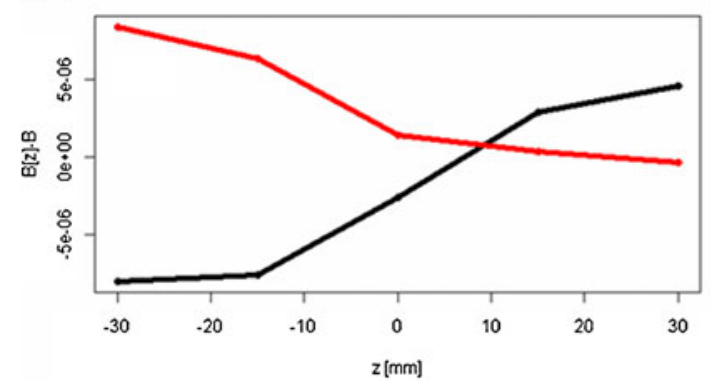

(b)

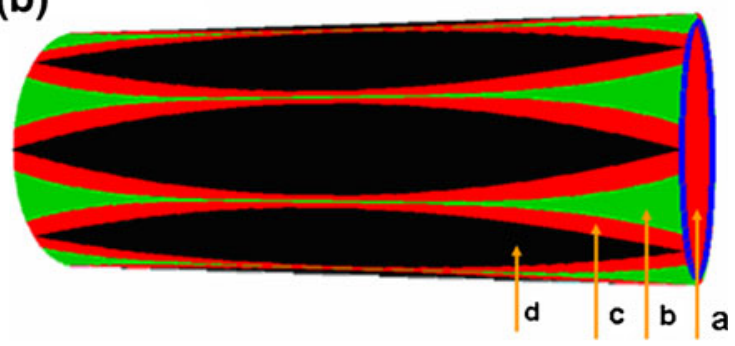

(d)

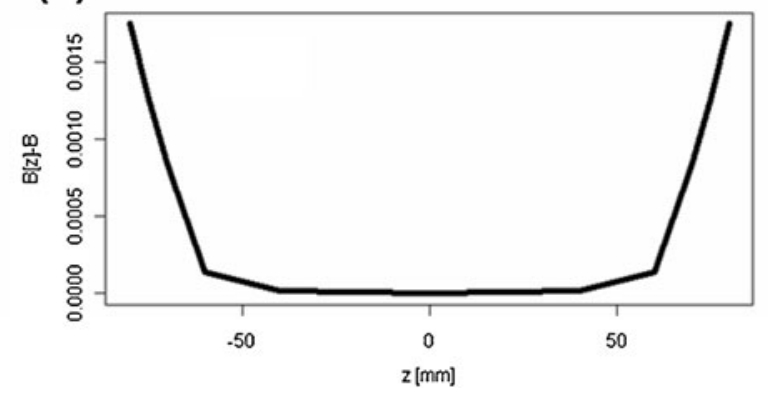

Figure 1. (a)- The ICR cell with dynamic harmonization [12] (a). Trapping electrodes with surface geometry close to spherical (b). Segments for electrostatic field harmonization (c). Grounded segments (d). Line separating detection electrodes assembly from excitation electrode assembly (b)- The designs of the compensation ICR cell with dynamic harmonization (a). Trapping electrodes with surface geometry close to spherical (b). Extra electrodes for compensation of magnetic field inhomogeneity by average radial electrostatic field (c). Segments for electrostatic field harmonization (d). Grounded segments electrodes are connected into groups for excitation and detection (not shown of the figure). (c)- Magnetic field near center for two 7 Tesla Bruker magnets. Black, installed in Bremen, red, installed in Moscow. Magnetic field [T]. (d)- Magnetic field for 7 Tesla Bruker magnet installed in Bremen. Magnetic field [T] 
free magnets with inhomogeneity of $100 \mathrm{ppm}$ in a cylindrical volume $25 \mathrm{~mm}$ in diameter and $40 \mathrm{~mm}$ in length. These instruments demonstrated the resolving power of about 100,000 for $\mathrm{m} / \mathrm{z}$ around 500. For such ICR mass spectrometers the inhomogeneity of the magnetic field is the main factor influencing the time of signal acquisition and resolving power. The inhomogeneity of the magnetic field was also the main limiting factor for an ICR mass spectrometer equipped with a 25 Tesla resistive magnet [16]. The inhomogeneity of the magnetic field in a $1 \mathrm{~cm}$ in diameter sphere was approximately $50 \mathrm{ppm}$ for this magnet. Correction of the magnetic field to achieve higher inhomogeneity is an expensive and complicated procedure.

Recently it was demonstrated that in the case of Gabrielse's type FT ICR cell, the influence of the inhomogeneity of the magnetic field could be decreased by compensating the electric field by accurately adjusting the compensation voltage on one of the electrodes of seven segment cell $[1,8,17]$.

Here the same idea is applied to the dynamically harmonized cell. We present a design of the ICR cell with magnetic field inhomogeneity compensation based on the principle of the dynamic field creation. Additional segments with a potential different from that on the main segments are introduced into the original ion trap with dynamic harmonization $[12,13]$, thus creating an additional electric field inside the cell. These segments are shaped by the curves of fourth order to z-coordinate (axial). Such electrodes can create a fourth order correction to the electric field and by turning voltage on them it is possible to compensate 2-nd order inhomogeneity of the magnetic field (see Figure 1b. Computer experiments were performed with additional segments shaped by curves of even higher orders: sixth and eighth to z-coordinate for correction of higher order magnetic field inhomogeneity.)

It was shown that by varying the voltage on these additional electrodes it is possible to make the disturbances of the cyclotron frequency from the magnetic field inhomogeneity independent of the z-oscillation amplitude. The inhomogeneity of the magnetic field for the two Bruker magnets is represented in Figure 1c, d. It can be seen that in a small region near the center the magnetic field has a mainly linear inhomogeneity and for a larger $\mathrm{z}$ the quadratic homogeneity dominates.

\section{Theory}

It was shown $[18,19]$ that only the inhomogeneity of the magnetic field in its $\mathrm{z}$ direction has a considerable influence on cyclotron frequency. The effects of the inhomogeneity in radial directions are negligible. Therefore for a single ion inside the FT ICR trap the general equation for radial force balance for simplified case of circular motion is:

$$
m \omega^{2} r=q B(r, z) v+q E_{r}(r, z)
$$

Where $\mathrm{m}$ - ion mass, $\mathrm{q}$ - ion charge, $B(r, z)$ is the intensity of magnetic field in the $\mathrm{z}$ direction, $E_{r}(r, z)$ - the radial component of the electric force formed by the ion trap, $\omega$ - the cyclotron frequency, $\mathrm{r}$ - cyclotron radius, $\mathrm{z}$ coordinate in the direction along the magnetic field, v velocity. Divided by the cyclotron radius this equation becomes:

$$
\frac{m \omega^{2}}{q} r=B(r, z) \omega+\frac{E_{r}(r, z)}{r}
$$

In case of the electric fields created by hyperbolic electrodes cyclotron frequency does not depend on $\mathrm{z}$. The dependence of the magnetic field $B(r, z)$ and the radial component of the electric field $E_{r}(r, z)$ on the $\mathrm{z}$ coordinate causes the cyclotron frequency dependence on the $\mathrm{z}$ coordinate. As a consequence ions with different amplitudes of $\mathrm{z}$ oscillation have different cyclotron frequencies and the ion cloud will experience dephasing during its cyclotron rotation. To prevent such dephasing the cyclotron frequency should be made independent of the $\mathrm{z}$ coordinate. Mathematically this means that its first derivative by $z$ is equal to zero. The first derivative of Equation 3 by the $\mathrm{z}$-coordinate is:

$$
\frac{m}{q} 2 \omega \omega_{z}^{\prime}=B_{z}^{\prime}(r, z) \omega+B(r, z) \omega_{z}^{\prime}+\left(\frac{E_{r}(r, z)}{r}\right)_{z}^{\prime}
$$

and by equalizing $\omega_{z}^{\prime}$ to zero we obtain:

$$
B_{z}^{\prime}(r, z) \omega+\left(\frac{E(r, z)}{r}\right)_{z}^{\prime}=0
$$

Taking into account that $\omega=\frac{q B}{m}$, we can rewrite Equation (5) in the following form:

$$
\frac{q}{m} B_{z}^{\prime}(r, z) B(r, z)+\left(\frac{E_{r}(r, z)}{r}\right)_{z}^{\prime}=0
$$

This is the equation describing the required relationship between the magnetic and the electric field in order for compensation to take place.

It is possible to describe the $\mathrm{z}$ component of the magnetic field for any magnet as a series of spherical functions as proposed in [19]:

$$
B_{z}=A_{1}^{0}+2 A_{2}^{0} z+3 A_{2}^{1} x+3 B_{2}^{1} y+3 A_{3}^{0}\left(2 z^{2}-x^{2}-y^{2}\right) / 2+\ldots
$$

Current shims of different geometry (circular, rectangular) are used for shimming different terms in expansion (7). Usually the main impact on the inhomogeneity of the magnetic field is caused by the quadratic term. 
As a consequence we simplified our task and considered only the inhomogeneity of the magnetic field in the following form:

$$
B=B_{0}\left(1+\gamma z^{2}\right)
$$

The effects of other terms were not included in our current considerations. This field may be corrected by the electric potential of the form:

$$
V=a+b\left(r^{2}-2 z^{2}\right)+c\left(8 z^{4}-24 z^{2} r^{2}+3 r^{4}\right)
$$

This is a result of electric field representation as a series of spherical harmonics. By inserting this into the Equation 6, we obtain:

$$
0=B_{0} \gamma 2 z \omega+\left(\frac{2 b r+c\left(-48 z^{2} r+12 r^{3}\right)}{r}\right)_{z}^{\prime}
$$

This equation can be satisfied for

$$
c=\frac{e B_{0}^{2} \gamma}{48 m}
$$

So, the quadratic term of the magnetic field inhomogeneity can be compensated by the fourth order spherical harmonics of the electric field.

The design of an ion trap capable to create such electric field is presented in Figure 1b. Additional segments shaped by the fourth order curve were introduced to the original ion trap with dynamic harmonization. The form of the curve obeys the equation:

$$
\alpha_{4}=\frac{2 \pi}{8} n \pm b_{0}\left(1-\left(\frac{z}{k * a}\right)^{4}\right)
$$

with $b_{0}=\frac{\pi}{7.2}-\frac{\pi}{60}, k=1.15$.

If the potential on the compensation electrodes is set equal to the potential of the housing and trapping electrodes then the compensated cell becomes similar to the original cell with dynamic harmonization. So the same cell design may be successfully used for magnets of different homogeneity of the magnetic field. For magnets of high homogeneity the potential on the compensation electrodes will be close to the potential on the housing electrodes.

In the original ion trap with dynamic harmonization [12] the trapping electrodes are shaped by following the equipotentials of harmonic field. In the proposed cell with compensation electrodes the position of the trapping electrodes remained the same. This means that when the potential on the compensation electrodes is not equal to the potential on the housing electrodes the trapping electrodes do not fit the equipotential of the compensated field. This leads to the presence of additional corrections of a higher order in the electrostatic field.

It is possible to create an exact averaged compensated field of the form (9) by segmenting the trapping electrodes. See Appendix I for more details.

\section{Computer Simulations}

Simulation of ion cloud dynamics in the cell with dynamic harmonization is a challenging problem. The time of transient duration for such a cell could reach $300 \mathrm{~s}$ [13]. During this time the ion accomplishes hundreds of millions rotations. As a consequence for such long times even slight numerical errors in the calculated electromagnetic field or in the integration of ion motion equations will lead to a considerable difference between computer simulation results and experiment.

For example recently performed computer simulations of ion cloud motion in the original ion trap with dynamic harmonization [12] showed a dephasing rate which was much faster than that observed experimentally. Further investigations showed that this dephasing occurred due to the dependence of the radial component of the electric force on the $\mathrm{z}$ coordinate. Such dependence occurred because of errors of the electrostatic potential calculations.

The potential distribution has been calculated by several methods: finite difference method (FDM) in cylindrical and Cartesian coordinates and finite element method (FEM). We performed a multi-grid successive over-relaxation with optimal parameter method for FDM in Cartesian coordinates and multigrid Gauss-Zeidel method for FDM in cylindrical coordinates. Seven-point stencil was used for approximation of the Laplasian. To obtain high accuracy the size of the mesh, number of intermediate meshes and number of iterations were varied. Also SIMION 8 (David Manura Scientific Instruments Services, Ringoes, NJ, USA) has been applied for comparison.

The accuracy of our calculations was controlled by comparing the analytically obtained averaged field with the field obtained for the case when the voltage on the compensation electrodes was equal to the voltage on the housing and trapping electrodes. The comparing procedure was the following. For the original ion trap with dynamic harmonization with radius $R$ and half length $Z$ the field averaged over the angle can be obtained as a solution for the system of equations:

$$
\begin{aligned}
& V(r, z)=a+b\left(r^{2}-2 z^{2}\right) \\
& V(R, 0)=\frac{\pi}{60} V \\
& V(R, Z)=V
\end{aligned}
$$

Cylindrical symmetry of the cell suggests that the field averaged by the angle of rotation must be the solution of the averaged boundary problem [12]. Here, $\mathrm{V}$ is the voltage on housing and trapping electrodes, and $\frac{\pi}{60}-$ the angle width of 
the housing electrode in the center of the cell. By solving this system of equations we can easily calculate the averaged by angle theoretical field. It is convenient to compare the obtained results with the field given by Equation (13) on the central axis because the averaging procedure may cause additional errors.

The results obtained for field accuracy using different methods are shown below. Simulations have been performed for a cell with the following dimensions [12]: radius of $28 \mathrm{~mm}$, half-length $-75 \mathrm{~mm}$, radius of the trapping electrode - $148.7 \mathrm{~mm}$. In the center of one of the trapping electrodes a circular hole of $6 \mathrm{~mm}$ in diameter for ion inlet was placed. We defined an error as the largest discrepancy between the numerically calculated field and the field given by (13) on the central axis.

- FDM Cylindrical coordinates - Error $=1.3 \%$. Mesh corresponds to 2 points per $1 \mathrm{~mm}$.

- FDM Cartesian coordinates - Error $=0.075 \%$. Mesh corresponds to 12 points per $1 \mathrm{~mm}$.

- FEM - Error $=0.65 \%$. Mesh corresponds to 2 points per $1 \mathrm{~mm}$.

- SIMION - Errors $0.56 \%$. Mesh corresponds to 10 points per $1 \mathrm{~mm}$.

For all methods different mesh sizes limited only by available computer memory and different number of iterations were tried.

Also, comparison of the solutions in the whole volume of the cell has been performed. The numerically obtained field potential was averaged by the angle and compared to the field given by Equation (13). All methods showed close accuracy: for radii less than $70 \%$ of the cell the error is about $1 \%$; for large radii the error is about $1.5 \%-2 \%$.

Additional details are placed in the Appendix II.

The other possible source of errors is integration of ion motion equations. This integration was performed using a fourth order Runge-Kutta method with frequency correction. Realization of the frequency correction was similar to the one used in the Boris integration method [20]. Time step of integration was chosen from the condition, that there are around 3000 calculation steps per one cyclotron period. For calculation of the electrostatic field inside the mesh element a trilinear interpolation method was used [21]. Also, numerous simulations in the hyperbolic field were performed in order to make sure that the integration procedure is not the source of errors.

To estimate the dephasing time of an ion cloud the following numerical experiment was carried out. The cyclotron motion during detection of ions with different $\mathrm{m} / \mathrm{q}$ in a $7 \mathrm{~T}$ magnetic field with different cyclotron radii and oscillation amplitudes was simulated.

The initial conditions for the equation of ion motion were the values of $\mathrm{z}$ coordinate, radius $\mathrm{r}$, and corresponding cyclotron velocity $\mathrm{v}$. The phase was the same for all of the experiments.
The z- oscillation amplitude was varied from $2 \mathrm{~mm}$ to $30 \mathrm{~mm}$ with $1 \mathrm{~mm}$ steps. Moments of ion intersection with the plane $x=0$ were recorded. Such method gives us the possibility to monitor the evolution of the cyclotron frequency.

The time of complete ion cloud dephasing is defined as the time corresponding to the moment in the cloud evolution when the head of cloud touches its tail. One rotation cycle for ions with different oscillation amplitudes takes different times. If one denotes the mean length of the cyclotron period for these ions as $\bar{t}$ and the standard deviation which corresponds to the ion cloud dephasing rate as $\Delta t$, then the number of rotations required for complete dephasing is $N_{\text {deph }}=\bar{t} / \Delta t$. And the time of dephasing is:

$$
T_{\text {deph }}=N_{\text {deph }} \bullet \bar{t}=\frac{\bar{t}^{2}}{\Delta t}
$$

Results of the simulations are presented in Figure 2.

The voltage on the compensation electrodes does not depend on the amplitude of ion oscillation in the potential well along the magnetic field (Figure 2c, d). Also no dependence on cyclotron radius was observed (Figure 2d, e, f).

An inversely proportional dependence of the optimal voltage on the compensation electrode from $\mathrm{m} / \mathrm{q}$ (Figure $2 \mathrm{a}$, $b, c)$ and a linear dependence from the value of inhomogeneity of the magnetic field were observed as predicted by theory.

For example, for an inhomogeneity coefficient $\gamma=4 \cdot 10^{-9}$ $\mathrm{mm}^{-2}$ the optimal compensation voltage is equal to $13 \mathrm{~V}$ for $\mathrm{m} / \mathrm{q}=300,8 \mathrm{~V}$ for $\mathrm{m} / \mathrm{q}=500$ and $6 \mathrm{~V}$ for $\mathrm{m} / \mathrm{q}=700$. The width at half height of the peaks on Figure 2 is equal to approximately $1 \mathrm{~V}$. This means that it can be expect that the proposed cell will effectively align the cyclotron frequency in an $\mathrm{m} / \mathrm{q}$ range of about $100 \mathrm{Da}$ for moderate $\mathrm{m} / \mathrm{q}$ and for the whole upper $\mathrm{m} / \mathrm{q}$ range.

The dependence of the dephasing time from the oscillation amplitude and radius, which can be seen in Figure 2, can be explained by numerical errors in the simulations of the electric field.

Simulations performed for the conventional ion trap with dynamic harmonization revealed an important rule that the accuracy of the electric field is the main factor influencing ion cloud dephasing [22].

It is also possible to compensate the linear ingomogeneity of the magnetic field using the proposed cell. For this it is necessary to set different potentials on the left and right compensation segments. The voltages on the compensation electrodes were changed in accordance with the following condition: $V_{l}+V_{r}=2 * V_{\text {trap }}$, where $V_{l, r}$ - are the voltages on left and right sets of compensation electrodes and $V_{\text {trap }}$ is the voltage on the housing electrodes. Thus the compensation voltages on the left and right 

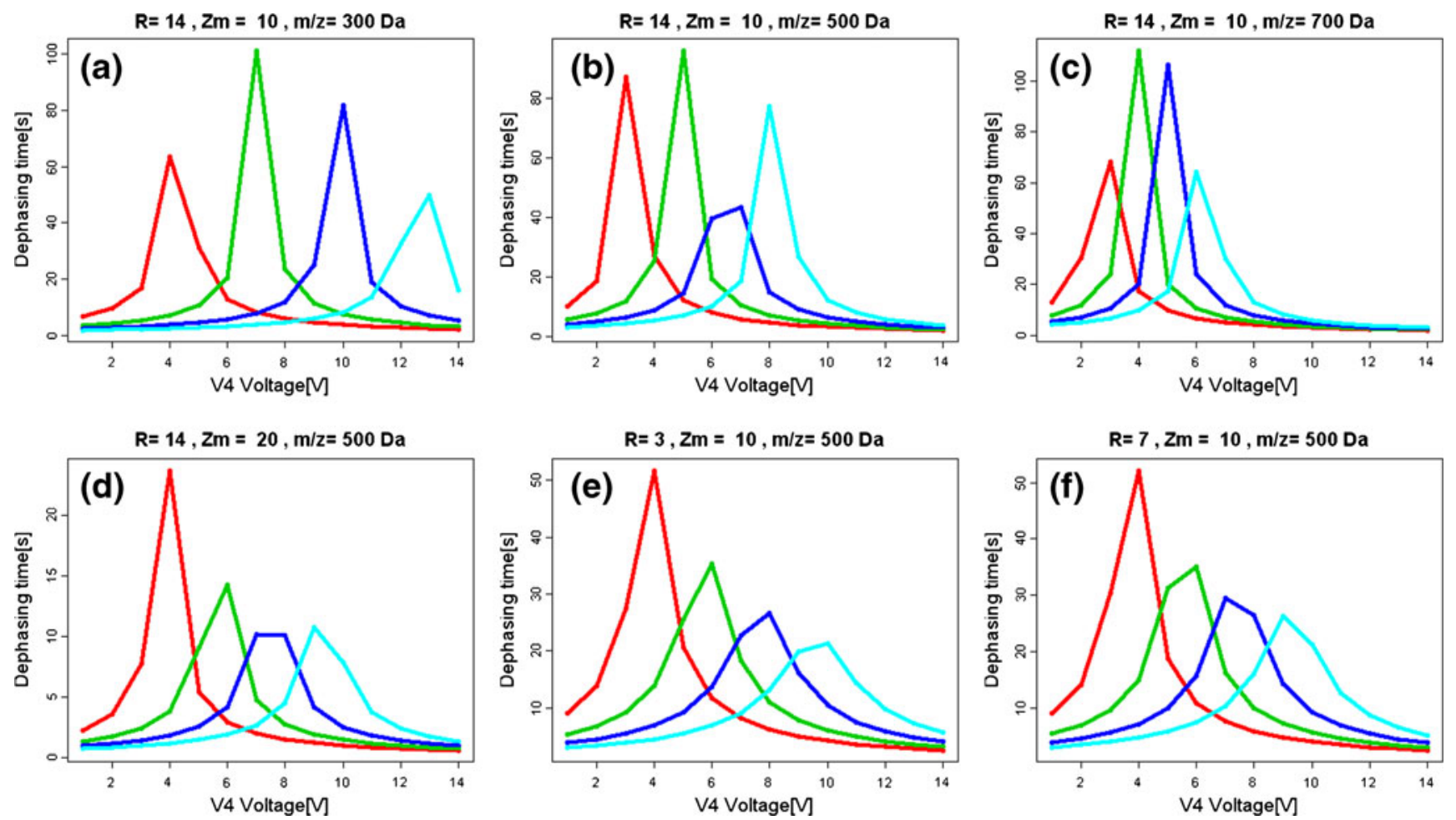

Figure 2. Dependence of the ion cloud dephasing time on the compensation electrode voltage for different values of the $z$ oscillation amplitude (zero to peak), radius and $\mathrm{m} / \mathrm{z}$. Red $\gamma=1 \cdot 10^{-9} \mathrm{~mm}^{-2}$, Green $\gamma=2 \cdot 10^{-9} \mathrm{~mm}^{-2}$, Blue $\gamma=3 \cdot 10^{-9} \mathrm{~mm}^{-2}$, Milky Blue $\gamma=$ $4 \cdot 10^{-9} \mathrm{~mm}^{-2}$. See Equation 8
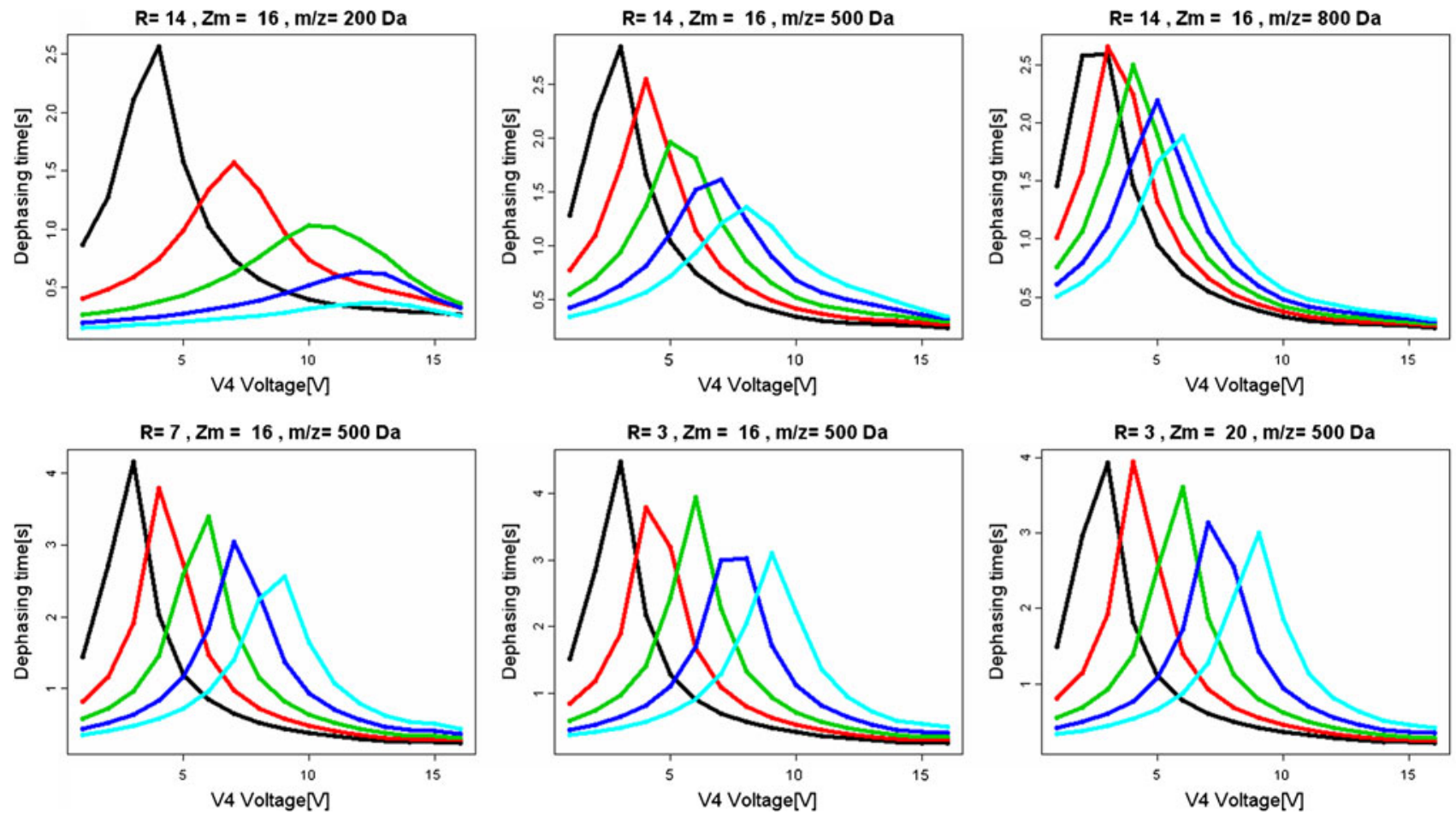

Figure 3. Dependence of the ion cloud dephasing time on the compensation electrode voltage for different values of the zoscillation amplitude (zero to peak), radius and $\mathrm{m} / \mathrm{z}$. Linear inhomogeneity. Time of synchronic motion vs. voltage on right set of compensation electrodes. $V_{l}+V_{r}=2^{\star} V_{\text {trap }} . B=B(1+\gamma z)$. Black $\gamma=1 \cdot 10^{-7} \mathrm{~mm}^{-1}$, Red $\gamma=3 \cdot 10^{-7} \mathrm{~mm}^{-1}$, Green $\gamma=5 \cdot 10^{-7} \mathrm{~mm}^{-1}$, Blue $\gamma=7 \cdot 10$ ${ }^{-7} \mathrm{~mm}^{-1}$, Milky Blue $\gamma=9 \cdot 10^{-7} \mathrm{~mm}^{-1}$ 

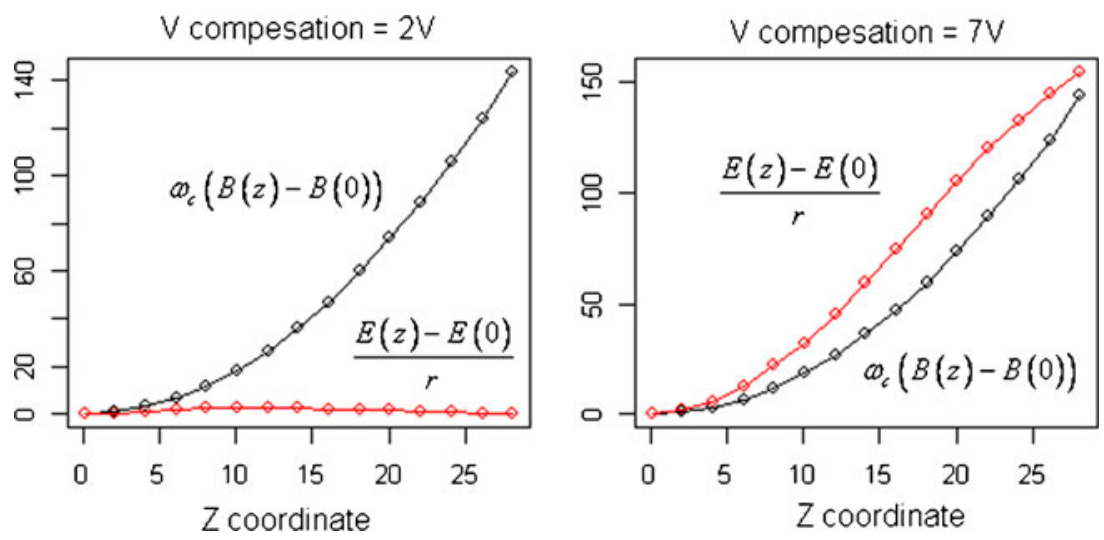

Figure 4. Compensation conditions for the case of $\gamma=2 \cdot 10^{-9} \mathrm{~mm}^{-2}, Z[\mathrm{~mm}] . \mathrm{B}[\mathrm{T}], \mathrm{w}\left[\mathrm{s}^{-1}\right], \mathrm{E} / \mathrm{r}\left[\mathrm{V} / \mathrm{m}^{2}\right]$

electrodes are symmetric with respect to the trapping voltage.

In Figure 3 the results of such compensation are shown. It can be seen that for linear inhomogeneity it is possible to correct the cyclotron frequency and increase the time of synchronous ion motion. Also the inverse proportionality of the dependence from $\mathrm{m} / \mathrm{q}$ and linear dependence from the inhomogeneity coefficient can be seen. The compensation works only for a certain $\mathrm{m} / \mathrm{q}$ range. The average order of such $\mathrm{m} / \mathrm{q}$ range is approximately hundreds of $\mathrm{Da}$. The complete compensation could be done for much narrower $\mathrm{m} / \mathrm{q}$ range (what is enough in case of fine structure resolution and isotopic patterns of proteins).

In order to compare these results with the compensation theory the following computational experiment was done. The $\mathrm{z}$ coordinates were frozen and the values of the magnetic field, electric force and current coordinates of the ions during their rotation were recorded. Approximately 150 records per cyclotron period were made.

For each $\mathrm{z}$ coordinate the mean radial component of the electric field $E_{r}(z)$ was calculated. The compensation theory predicts that in order for compensation to occur, (as follows from Equation 6) the following condition must be met:

$$
\omega_{c} B_{z}^{\prime}(z)+\frac{E_{z}^{\prime}(z)}{r}=0
$$

If derivative $E_{z}^{\prime}(z)$ is replaced with a finite difference $\Delta E(z)=\frac{E(z)-E(0)}{z}$ and similar is done for $B_{z}^{\prime}(z)$, it may be concluded that curves $\omega_{c}(B(z)-B(0))$ and $\frac{z(z)-E(0)}{r}$ should match in order to meet the compensation condition. From Figure 4 it can be seen that by adjusting the compensation electrode voltage one can almost satisfy these conditions.

It can be clearly seen that for each $\mathrm{z}$ coordinate the inhomogeneity of the magnetic field was compensated by a correction in the electric force.

\section{Conclusions}

The theory of compensation of the magnetic field inhomogeneity inside an FT ICR cell with dynamic harmonization by introducing specific electric field corrections is presented. An ICR cell design is proposed in which the inhomogeneous component of the magnetic field of the second order is compensated by an electric field, created by incorporated into the housing electrode assembly special electrodes which borders are shaped by a 4-th order curve. By setting different voltages on left and right set of compensation electrodes, it also possible to compensate a linear inhomogeneity. Computer simulations have shown that in the proposed cell design the inhomogeneity of the magnetic field can be effectively compensated in relatively large mass to charge ratio range and a considerable increase in the resolving power in the case of low homogeneity of the magnetic field could be obtained.

\section{Acknowledgments}

The authors acknowledge support for this work by FASIE grant No. $9988 \mathrm{p} / 16759$.

The FDM method was implemented by Ivan Tsibulin using the Ani-3D software developed by Victor Vasilevskii. The authors thank Sergei Bogomolov and Vadim Anrdeev for fruitful discussions and useful suggestions on the calculation of the electrostatic field, and Anton Grigoriev for his help in its realization. The authors acknowledge the support from the Russian Foundation of Basic Research (grant 10-04-13306), from the Russian Federal Program (state contracts 14.740.11.0755, 16.740.11.0369), and from the Fundamental Sciences for Medicine Program of the Russian Academy of Sciences and from Bruker company.

\section{Appendix I}

The idea to crate a cell providing an averaged field Equation 9 is based on the principle of a dynamic field. A schematic design is presented on Figure 5. Only the 
second and fourth corrections of the electric field were considered. The consideration of higher harmonics is the same. Instead of a circular trapping electrode a segmented flat trapping electrode was proposed. The cylindrical surface is the same as in the original cell with dynamic harmonization. The trapping electrode is flat but is segmented into sectors by curves of the second and fourth order. The schematic design of the cell does not include gaps between electrodes. Also for the case of simplicity the width of housing electrodes in the center of the cell considered to be zero.

To determine the relationship between the voltages and parameters of the field Equation (9), the field must be averaged by angle. Using definitions from Figure 5 on the cylindrical surface one can obtain:

$$
\begin{gathered}
V(R, z)=a+b\left(R^{2}-2 z^{2}\right)+c\left(8 z^{4}-24 z^{2} R^{2}+3 R^{4}\right)= \\
\frac{N}{\pi}\left(V_{4} \frac{\frac{\pi}{N}}{L^{4}} z^{4}+V_{2}\left(\frac{\frac{\pi}{N}}{L^{2}} z^{2}-\frac{\pi}{L^{4}} z^{4}\right)+V_{0}\left(\frac{\pi}{N}-\frac{\frac{\pi}{N}}{L^{2}} z^{2}\right)\right)
\end{gathered}
$$

An averaged field on the trapping electrode:

$$
\begin{array}{r}
V(r, L)=a+b\left(r^{2}-2 L^{2}\right)+c\left(8 L^{4}-24 L^{2} r^{2}+3 r^{4}\right)= \\
\frac{N}{\pi}\left(V^{\prime}{ }_{4} \frac{\frac{\pi}{N}}{R^{4}} r^{4}+V_{2}^{\prime}\left(\frac{\frac{\pi}{N}}{R^{2}} r^{2}-\frac{\frac{\pi}{N}}{R^{4}} r^{4}\right)+V_{0}^{\prime}\left(\frac{\pi}{N}-\frac{\frac{\pi}{N}}{R^{2}} r^{2}\right)\right)
\end{array}
$$

By equalizing Equations (16) and (17), the following system of equations for the potentials on the electrodes can be obtained:

$$
\left\{\begin{array} { l } 
{ \frac { ( V _ { 4 } - V _ { 2 } ) } { L ^ { 4 } } = 8 c } \\
{ \frac { ( V _ { 2 } - V _ { 0 } ) } { L ^ { 2 } } = - 2 b - 2 4 c R ^ { 2 } } \\
{ V _ { 0 } = a + b R ^ { 2 } + 3 c R ^ { 2 } }
\end{array} \quad \left\{\begin{array}{l}
\frac{\left(V_{4}{ }^{\prime}-V_{2}{ }^{\prime}\right)}{R^{4}}=3 c \\
\frac{\left(V_{2}{ }^{\prime}-V_{0}{ }^{\prime}\right)}{R^{2}}=b-24 c L^{2} \\
V_{0}{ }^{\prime}=a-2 b L^{2}+8 c L^{4}
\end{array}\right.\right.
$$

As can be seen by solving this system of equations it is possible to adjust the potentials on the electrodes to create a field of the exact form (Equation (9)). The same technique is applicable to create a field of any other cylindrically symmetric form by introducing additional segments shaped by curves of higher order.

\section{Appendix II}

In this section a detailed discussion of the calculations of the electrostatic field are presented.

Several different methods were applied to obtain a very high accuracy in the procedure of the electric field simulation. The simplest one is the FDM method in a Cartesian coordinate system. The main disadvantage of this method is the error of approximation of the electrodes on the mesh. A simple shift method [23] was used for approximation the boundary conditions, so the approximation error is of
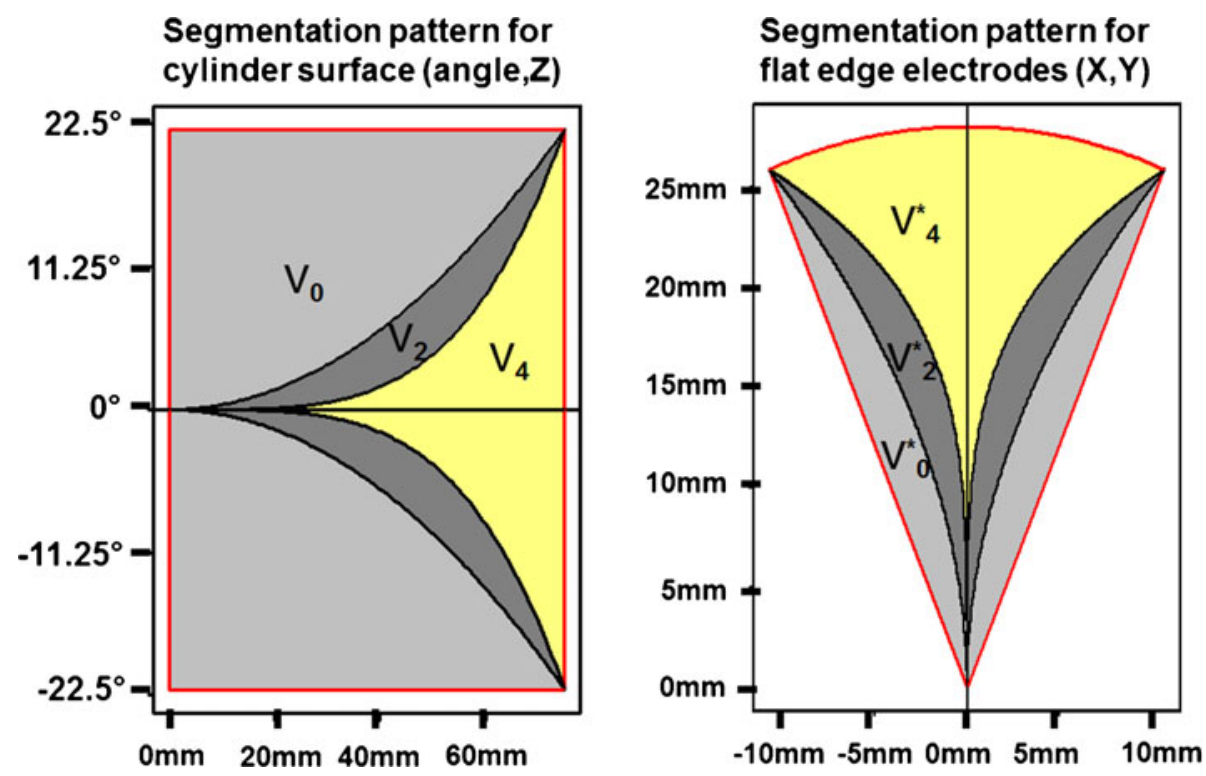

Figure 5. The schematic design of the ion trap with dynamic harmonization capable to create exact field of form (9). Segmentation pattern for cylindrical surface and trapping electrode for $N=8$ segment cell. Variables $V_{0}, V_{2}, V_{4}$ are voltages applied to segments on cylindrical surface. Variables $V_{0}^{\star}, V_{2}^{\star}, V_{4}^{\star}$ are voltages applied to segments on flat trapping electrode. Segments of the cell are shaped by curves of second and fourth order 


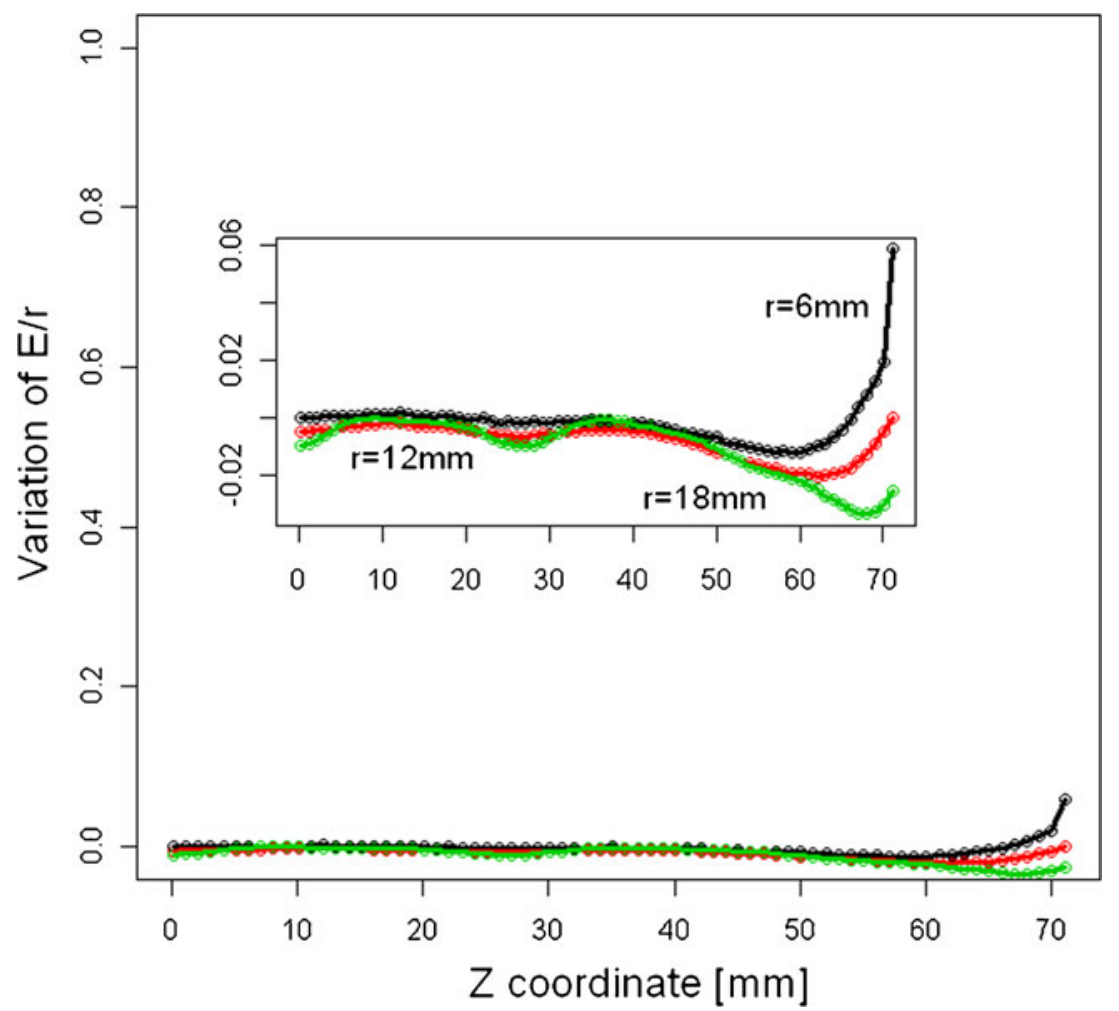

Figure 6. The dependence of radial component of electric force in original ion trap with dynamic harmonization on $z$ for different radii. $\frac{E_{r}(z) / r}{E_{r_{1}}(0) / r_{1}}-1, r_{1}=6 \mathrm{~mm}$

the order of the mesh size. But the important advantage is that the mesh is uniform and iterative methods for solving the boundary problem can converge very fast. A seven-point stencil was used for approximating the Laplace operator on the mesh, and also experiments with a 19-point stencil [24] were carried out and no considerable difference was found.
The electrostatic potential was calculated in the sector $\varphi \in[0,(\pi / 2)]$ on a uniform mesh. If the mesh approximation of the potential on a mesh point with indexes $x_{n}, y_{m}, z_{k}$ is denoted as $u_{n, m, k}$, then one step of the numerical solution was setting the value in this point as follow [25]:

$$
u_{n, m, k}=(1-w) u_{n, m, k}+w\left(\frac{u_{n-1, m, k}+u_{n+1, m, k}+u_{n, m-1, k}+u_{n, m+1, k}+u_{n, m, k-1}+u_{n, m, k+1}}{6}\right)
$$

Where parameter $\mathrm{w}$ is defined as:

$$
w=\frac{2}{1+\sqrt{2-2\left(\sin ^{2}\left(\frac{\pi}{2 N}\right)+\sin ^{2}\left(\frac{\pi}{2 M}\right)+\sin ^{2}\left(\frac{\pi}{2 K}\right)\right)}}
$$

And N, M, K - are the maximal numbers of points on the mesh in the $\mathrm{x}, \mathrm{y}$ and $\mathrm{z}$ directions.

One step of numerical solving a boundary problem is applying formula (19) for all grid points. This is a well known successive over-relaxation method with optimal parameter $[23,25]$. In order to increase the convergence a multi grid method was used. First the solution was obtained on a rough grid and then this solution was used as the initial condition for an iterative method on a fine grid. Different ways of choosing intermediate grids and different numbers of iteration on them were used.

One way to escape approximation errors is by implementing FDM in cylindrical coordinates. In addition rotation symmetry allows to considerably save computer memory by solving the problem only in the region $\varphi \in\left[0,\left(\pi / N_{\mathrm{sec}}\right)\right]$, where $N_{\text {sec }}$ is the number of sectors. As a consequence it is possible to use finer meshes with more points in them. For electrode approximation a simple shift method was used. The approximation of electrodes on cylinder surface does not contain errors except for the region around shaping curve. And the trapping electrodes are still approximated with errors of the order of mesh size. 
A Gauss-Zeidel iterative method was used to solve a system of algebraic equations. If the grid approximation of the potential value in a certain point of the grid with indexes $r_{n}, \varphi_{m}, z_{k}$ is denoted as $u_{n, m, k}$ then one step of the numerical solution was setting the value in this point as follow [23]:

$$
\begin{aligned}
u_{n, m, k}= & \frac{\beta}{2+4 \beta} \\
& \left(\begin{array}{c}
n^{2}\left(u_{n-1, m, k}+u_{n+1, m, k}\right)+\frac{n}{2}\left(u_{n+1, m, k}-u_{n-1, m, k}\right)+ \\
+\frac{1}{\beta}\left(u_{n, m-1, k}+u_{n, m+1, k}\right)+n^{2}\left(u_{n, m, k-1}+u_{n, m, k+1}\right)
\end{array}\right)
\end{aligned}
$$

Here $\beta=\frac{\pi^{2}}{N_{S_{e c}{ }^{2} M}}, N_{\mathrm{sec}}$ is the number of sectors (eight in our case), and $\mathrm{M}$ - maximal number of points of angle discretization. One cycle of the calculations (iteration) is applying expression (Equation 21) to all mesh points. To increase the convergence a multi grid method was used

But approximation of the trapping electrodes still contains errors. We did not succeed in obtaining high accuracy using this method because iterative methods did not converge for large meshes. But in case of small meshes the solution was more accurate, as compared to the one obtained for FDM in Cartesian coordinates with equal mesh size.

A method that does not have approximation errors is FEM. In addition to the surface elements corresponding to electrode boundary especial curved cylindrical elements were used. But the accuracy of this method also was not very high.

The best results were obtained using FDM in Cartesian coordinates for very fine mesh. On Figure 6, the variation of the ratio $E_{r}(z) / r$ averaged over the angle along the central axis is presented. In an ideal cell the ratio $E_{r}(z) / r$ should be independent of both $\mathrm{z}$ and $\mathrm{r}$. But because of the calculation errors in the electrostatic field certain dependence is observed.

It can be seen that the variation of $E_{r}(z) / r$ considerably depends on the accuracy of the calculated electric field. The radial component of the electric field is the derivative of the electric potential by the radius and derivation introduces additional errors. The field from a rectangular mesh was interpolated to a cylindrical and then a four-point derivative was used to obtain the electric force in the radial direction.

\section{References}

1. Kaiser, N.K., Savory, J.J., McKenna, A.M., Quinn, J.P., Hendrickson, C.L., Marshall, A.G.: Electrically compensated Fourier transform ion cyclotron resonance cell for complex mixture mass analysis. Anal. Chem. 83(17), 6907-6910 (2011)

2. Marshall, A.G., Hendrickson, C.L., Jackson, G.S.: Fourier transform ion cyclotron resonance mass spectrometry: a primer. Mass Spectrom Rev. 17(1), 1-35 (1998)

3. Bogdanov, B., Smith, R.D.: Proteomics by FTICR mass spectrometry: top down and bottom up. Mass Spectrom. Rev. 24(2), 168-200 (2005)

4. Marshall, A.G., Rodgers, R.P.: Petroleomics: the next grand challenge for chemical analysis. Acc. Chem. Res. 37(1), 53-59 (2004)
5. Kim, S., Kramer, R.W., Hatcher, P.G.: Graphical method for analysis of ultrahigh-resolution broadband mass spectra of natural organic matter, the Van Krevelen diagram. Anal. Chem. 75, 5336-5344 (2003)

6. Nikolaev, E.N., Jertz, R., Grigoryev, A., Baykut, G.: Fine structure in isotopic peak distributions measured using a dynamically harmonized Fourier transform ion cyclotron resonance cell at 7 T. Anal. Chem. 84 (5), 2275-2283 (2012)

7. Gabrielse, G., Haarsma, L., Rolston, S.L.: Open-endcap Penning traps for high precision experiments. Int. J. Mass Spectrom. Ion Processes 88, 319-332 (1989)

8. Brustkern, A.M., Rempel, D.L., Gross, M.L.: An electrically compensated trap designed to eighth order for FT-ICR mass spectrometry. $J$. Am. Soc Mass Spectrom 19(9), 1281-1285 (2008)

9. Nikolaev, E.N., Heeren, R.M.A., Popov, A.M., Pozdneev, A.V., Chingin, K.S.: Realistic modeling of ion cloud motion in a Fourier transform ion cyclotron resonance cell by use of a particle-in-cell approach. Rapid Commun. Mass Spectrom. 21, 3527-3546 (2007)

10. Nikolaev, E.N., Miluchihin, N., Inoue, M.: Evolution of an ion cloud in a Fourier transform ion cyclotron resonance mass spectrometer during signal detection: its influence on spectral line shape and position. Int. J. Mass Spectrom. Ion Processes 148(3), 145-157 (1995)

11. Vladimirov, G., Hendrickson, C.L., Blakney, G.T., Marshall, A.G., Heeren, R.M., Nikolaev, E.N.: Fourier transform ion cyclotron resonance mass resolution and dynamic range limits calculated by computer modeling of ion cloud motion. J. Am. Soc Mass Spectrom. 23 (2), 375-384 (2012)

12. Boldin, I.A., Nikolaev, E.N.: Fourier transform ion cyclotron resonance cell with dynamic harmonization of the electric field in the whole volume by shaping of the excitation and detection electrode assembly. Rapid Commun. Mass Spectrom. 25, 122-126 (2011)

13. Nikolaev, E.N., Boldin, I.A., Jertz, R., Baykut, G.: Initial experimental characterization of a new ultra-high resolution FTICR cell with dynamic harmonization. J. Am. Soc. Mass Spectrom. 22(7), 1125-1133 (2011)

14. Vladimirov, G., Kostyukevich, Y., Marshall, A.G., Hendrickson, C.L., Blakney, G.T., Nikolaev, E.N. Influence of different components of magnetic field inhomogeneity on cyclotron motion coherence at very high magnetic field Proceedings of the 58th ASMS Conference on Mass Spectrometry and Allied Topics; Salt Lake City, UT, May (2010)

15. Vilkov, A.N., Gamage, C.M., Misharin, A.S., Doroshenko, V.M., Tolmachev, D.A., Tarasova, I.A., Kharybin, O.N., Novoselov, K.P., Gorshkov, M.V.: Atmospheric pressure ionization permanent magnet Fourier transform ion cyclotron resonance mass spectrometry. J. Am. Soc. Mass Spectrom. 18(8), 1552-1558 (2007)

16. Shi, S.D.-H., Drader, J.J., Hendrickson, C.L., Marshall, A.G.: Fourier transform ion cyclotron resonance mass spectrometry in a high homogeneity 25 Tesla resistive magnet. J. Am. Soc. Mass Spectrom. 10, 265-268 (1999)

17. Tolmachev, A.V., Robinson, E.W., Wu, S., Kang, H., Lourette, N.M., Paša-Tolić, L., Smith, R.D.: Trapped-ion cell with improved DC potential harmonicity for FT-ICR MS. J. Am. Soc. Mass Spectrom. 19 (4), 586-597 (2008)

18. Laukien, F.H.: The effects of residual spatial magnetic field gradients on Fourier transform ion cyclotron resonance spectra. Int. J. Mass Spectrom. Ion Processes 73, 81-107 (1986)

19. Anderson, W.A.: Electrical current shims for correcting magnetic fields. Rev. Sci. Instrum. 32, 241-250 (1961)

20. Boris, J.P. The acceleration calculation from a scalar potential. Plasma Physics Laboratory: Princeton University, MATT-152, March (1970)

21. Kang, H.R.: Computational color technology. SPIE PRESS Bellingham, Washington (2006)

22. Kostyukevich Y. Nikolaev E. Studying of ion cloud dephising in FT ICR trap with dynamic harmonization. Proceedings of the 60th ASMS Conference on Mass Spectrometry and Allied Topics; Vancouver, Canada, May (2012)

23. Samarskii, A.A.: Theory of difference schemes. Nauka, Moscow (Russian) (1989)

24. O’Reilly, R.C., Beck, J.M. A family of large-stencil discrete Laplacian approximations in three-dimensions. Int. J. Numer. Methods Eng. 1-16 (2006)

25. Samarskii, A.A., Andreev, V.B.: The Different Methods For Elliptic Equations. Nauka, Moscow (Russian) (1976) 